\title{
Cost-effectiveness of telehealth for patients with depression: evidence from the Healthlines randomised controlled trial
}

Padraig Dixon, Sandra Hollinghurst, Louisa Edwards, Clare Thomas, Alexis Foster, Ben Davies, Daisy Gaunt, Alan A. Montgomery and Chris Salisbury

\section{Background}

Depression is a prevalent long-term condition that is associated with substantial resource use. Telehealth may offer a costeffective means of supporting the management of people with depression.

\section{Aims \\ To investigate the cost-effectiveness of a telehealth} intervention ('Healthlines') for patients with depression.

\section{Method}

A prospective patient-level economic evaluation conducted alongside a randomised controlled trial. Patients were recruited through primary care, and the intervention was delivered via a telehealth service. Participants with a confirmed diagnosis of depression and PHQ-9 score $\geq 10$ were recruited from 43 English general practices. A series of up to 10 scripted, theory-led, telephone encounters with health information advisers supported participants to effect a behaviour change, use online resources, optimise medication and improve adherence. The intervention was delivered alongside usual care and was designed to support rather than duplicate primary care. Cost-effectiveness from a combined health and social care perspective was measured by net monetary benefit at the end of 12 months of follow-up, calculated from incremental cost and incremental quality-adjusted life years (QALYS). Cost-consequence analysis included cost of lost productivity, participant out-of-pocket expenditure and the clinical outcome.

\section{Results}

A total of 609 participants were randomised -307 to receive the Healthlines intervention plus usual care and 302 to receive usual care alone. Forty-five per cent of participants had missing quality of life data, $41 \%$ had missing cost data and $51 \%$ of participants had missing data on either cost or utility, or both. Multiple imputation was used for the base-case analysis. The intervention was associated with incremental mean per-patient National Health Service/personal social services cost of $£ 168$ (95\% Cl $£ 43$ to $£ 294)$ and an incremental QALY gain of $0.001(95 \% \mathrm{Cl}-0.023$ to 0.026$)$. The incremental cost-effectiveness ratio was $£ 132630$. Net monetary benefit at a cost-effectiveness threshold of $£ 20000$ was $-£ 143(95 \% \mathrm{Cl}-$ $£ 164$ to $-£ 122$ ) and the probability of the intervention being cost-effective at this threshold value was 0.30 . Productivity costs were higher in the intervention arm, but out-of-pocket expenses were lower.

\section{Conclusions}

The Healthlines service was acceptable to patients as a means of condition management, and response to treatment after 4 months was higher for participants randomised to the intervention. However, the positive average intervention effect size was modest, and incremental costs were high relative to a small incremental QALY gain at 12 months. The intervention is not likely to be cost-effective in its current form.

\section{Declaration of interest}

None.

\section{Copyright and usage}

(C) The Royal College of Psychiatrists 2016. This is an open access article distributed under the terms of the creative Commons Attribution (CC BY) licence.
The management of long-term conditions is challenging. Some $30 \%$ of the population of England are estimated to have a longterm condition, ${ }^{1}$ whereas their treatment constitutes $70 \%$ of all health and care expenditure in England. ${ }^{1}$ Depression may be considered as an exemplar long-term condition: it is prevalent, ${ }^{2}$ recurrent, ${ }^{3}$ associated with morbidity and comorbidity, ${ }^{4}$ mortality ${ }^{4}$ and substantial resource use. ${ }^{5}$

One form of care that may be relevant to the management of depression (and other long-term conditions) is telehealth. ${ }^{6}$ Telehealth can be defined as the use of technology to deliver healthcare remotely and to promote patient self-management. ${ }^{7}$ A multicentre, parallel, two-arm, individually randomised controlled trial (RCT) was undertaken to assess the effectiveness and cost-effectiveness of a structured, theory-driven telehealth intervention - 'Healthlines' - for patients with depression. This paper describes the results of an economic evaluation conducted alongside the RCT in order to estimate the cost-effectiveness of the Healthlines service for primary care patients with depression.

\section{Method}

Patients with a confirmed diagnosis of depression, and a score $\geq 10$ on the validated ${ }^{8}$ and widely used ${ }^{9}$ nine-item Patient Health Questionnaire (PHQ-9) depression scale ${ }^{10}$ were recruited from 43 general practices in or near the English cities of Bristol, Sheffield and Southampton. The design of the telehealth intervention, ${ }^{11}$ the protocol for the $\mathrm{RCT}^{12}$ and results of the $\mathrm{RCT}^{13,14}$ have been reported elsewhere. In brief, 609 patients were individually randomised to receive either usual care or usual care plus the Healthlines service. Patients were followed up for 12 months.

The main perspective adopted for the economic evaluation, in line with National Institute for Health and Care Excellence (NICE) guidance, ${ }^{15}$ was that of the health and social care provider. A cost-effectiveness analysis was carried out from this perspective comparing costs, to the UK National Health Service (NHS) and personal social services (PSS) sectors, with quality-adjusted life years (QALYs). We also separately report the impact of the 
intervention on personal expenditure and on productivity as part of a cost-consequence analysis.

\section{Intervention}

The Healthlines service was developed based on a programme of work ${ }^{13}$ that encompassed a systematic review of effective telehealth interventions, ${ }^{16}$ qualitative work on patient and provider experiences of telehealth, ${ }^{17}$ a survey of patient attitudes toward telehealth ${ }^{18}$ and reviews of evidence-based treatment guidelines for depression. This work informed the development and delivery of the de novo telehealth intervention ${ }^{11}$ delivered in the RCT.

The intervention consisted of telephone support designed to encourage participants to use resources available from the internet, along with efforts to optimise treatment and promote medication adherence. Resources available included a self-directed cognitive-behavioural therapy (CBT) course involving the use of a book or the CBT website 'Living Life to the Full Interactive' (LLTTFi); access to a secure web portal which included health information; access to the Big White Wall (BWW) online forum; requests for discussions with the trial's trained health information advisors (HIAs - see below); and copies of letters sent to the participant's general practitioner (GP).

Scripted, scheduled telephone encounters took place over the 12 months of trial follow-up between trial participants and HIAs. The HIAs, non-clinical staff with experience in providing health information to members of the public by telephone, were provided with additional training in order to deliver the Healthlines service. They were employed by the NHS, and they received supervision from nursing staff and from pharmacists. Participants randomised to the intervention were eligible to receive a maximum of 10 such telephone-based encounters and also receive usual care.

Participants randomised to the control arm received usual care for depression.

\section{Measurement of outcomes}

The primary clinical outcome for the trial was the proportion of patients responding to treatment at 4 months from randomisation, measured using the PHQ-9 questionnaire. Response was defined as a reduction (from baseline) in PHQ-9 of at least 5 points and an overall score of less than 10. PHQ-9 responses were also collected at 8 and 12 months.

Information on health-related quality of life was collected at baseline, 4, 8 and 12 months using the EQ-5D-5L questionnaire, ${ }^{19}$ a standardised, generic instrument that allows for the measurement and valuation of health-related quality of life. The EQ-5D-5L measures five dimensions of health-related quality of life (mobility, self-care, usual activities, pain and discomfort, and anxiety and depression) with five categories of health states corresponding to no problems, slight problems, moderate problems, severe problems and extreme problems. The EQ-5D-3L version of the questionnaire is known to discriminate between different levels of depression severity ${ }^{20}$ and has 'adequate' validity and responsiveness in patients with depression. ${ }^{21}$

\section{Measurement and valuation of resource use}

With participant consent, details of all primary care consultations and relevant prescriptions (antidepressants, hypnotics and anxiolytics, and drugs used in psychoses and related disorders) issued during the 12-month follow-up period were extracted from GP records.

Questionnaires administered at 4,8 and 12 months requested information on other healthcare and social care accessed for reasons connected with the participant's depression. This questionnaire, versions of which have been used in previous withintrial primary care evaluations, is available from the 'Database of
Instruments for Resource Use Measurement' at www.dirum.org. The questionnaire included the use of hospital services, attendance at accident and emergency departments, ambulance use, and the use of PSS such as social worker services. The questionnaires also requested information on the use of private therapies, out-ofpocket expenditure, time-off from work, benefits received, informal care and the use of voluntary-sector services. The questionnaires could be completed online or by post, but those completing them online still had to complete the EQ-5D by post because of licencing restrictions.

All resources were valued and costs reported in pound sterling at $2012 / 13$ prices. Curtis ${ }^{22}$ was used to cost primary care consultations, community health and social care where possible. NHS National Reference Costs for $2012 / 13^{23}$ were used to value hospital and ambulance use. All sources are described in the supplementary material (see in particular Tables DS1 and DS2). The costs of drugs prescribed in primary care and reported in medical records were based on the Prescription Cost Analysis England database ${ }^{24}$ and cross-checked against the British National Formulary. The value of personal expenditure was obtained directly from participant responses to questionnaires.

Productivity costs were calculated from reports of working days missed by trial participants, by friends and relatives as a consequence of the participant's depression, and time from work to attend healthcare appointments. All of this time was valued at the national median gross hourly wage of $£ 11.59$ for 2013 . $^{25}$

Data on the resources used to provide the Healthlines service were extracted from provider computer systems. This included the number and length of all telephone calls, the number of attempts by a HIA to contact a participant, the use of the LLTTFi website, the number of people who were sent the designated CBT book, and the use of the BWW website. Resources involved in establishing the service included training costs and the purchase of licences for LLTTFi and BWW, and these were included in the calculation of intervention cost. Table DS3 in the online supplementary material summarises the unit costs of these resources.

The HIAs were remunerated at Band 4 of the NHS 'Agenda for Change' pay scale. Cost-per-hour was estimated using the framework of Curtis, ${ }^{22}$ adjusted to reflect the working pattern of the HIAs. Anonymised task-scheduling diaries kept by the HIAs were used to estimate the proportion of their time spent not in contact with intervention participants: the ratio of contact/noncontact time was estimated to be 2:1. Further adjustment was made to allow for the 40-h workweek of the HIAs. HIAs received initial and ongoing training from a nurse-grade trainer and from a consultant psychiatrist in the LLTTFi package. The costs of training were amortised over an assumed duration of 3 years. This reflects the consideration that any training received would be relevant to the service for at least 3 years, after which additional training might become necessary. We regard this as a conservative assumption.

\section{Analysis of data}

All analyses were conducted in Stata 13.1 (Statacorp: College Station, Texas).

The distribution of all data used in the analysis was inspected and summary measures such as means and standard deviations calculated. The data were inspected for missingness. Data relating to the use of the intervention and primary care data (including prescriptions) were generally complete. Less than $0.5 \%$ of data relating to primary care consultations were missing. These missing values were imputed with mean values at the participant level. The resulting data-set formed our 'available cases'. The predominant sources of any remaining missing data were questionnaire 
responses, particularly questions relating to resource use and quality of life. In spite of satisfactory data completeness on primary care and the intervention, missing questionnaire responses meant that $45 \%$ of participants had missing quality of life data, $41 \%$ had missing cost data, and $51 \%$ of participants had missing data on either cost data or utility data, or both. Complete cases had complete data on all cost items and all quality of life data items at all time points, and $49 \%$ of participants ( $47 \%$ in the intervention arm, $51 \%$ in the control arm) qualified as complete cases under this definition.

Missing data were imputed using multiple imputation by chained equations, as implemented by the - ice - command ${ }^{26,27}$ in Stata 13.1. Data were assumed to be missing at random (MAR). The imputation model was stratified by trial $\operatorname{arm}^{27}$ and included demographic and cost variables without missing data alongside clinical outcome variables at baseline and follow-up, cost and utility variables with missing data, depression history, baseline depression status and whether patients were being prescribed antidepressants. Predictive mean matching was used to account for non-normal distributions in some included variables. ${ }^{27}$ Passive imputation was performed for categorical variables, such as binary PHQ-9 scores, that were functions of imputed variables.

Costs were imputed at the level of the major aggregate costs (e.g. primary care, medication, PSS costs and other NHS costs). The number of imputations ( $n=60)$ was selected to be greater than the proportion of missing data following White et al. ${ }^{27}$

Health-related quality of life utility measures at each follow-up time point were imputed, which were then used to generate QALY estimates. Utility values were calculated at baseline and the three follow-up time points. These data were obtained from participant responses to the EQ-5D-5L. We used the Euroqol UK crosswalk value set for mapping responses to the three-level version of EQ$5 \mathrm{D} .^{28}$ QALYs were calculated from the utility data using the 'area under-the-curve' method and adjusted for baseline differences in EQ-5D-5L scores. ${ }^{29}$

Cost-effectiveness analysis was performed on the imputed dataset using the methods described in Faria et $a l^{30}$ which implement 'Rubin's rules', ${ }^{31}$ in order to reflect the variation within and between the 60 imputed data-sets. Regression analysis was used to generate the cost-effectiveness results and to characterise the uncertainty surrounding point estimates. Seemingly unrelated regression (SUR) was used to jointly model costs and QALYs using the - sureg - command in Stata. Baseline imbalances in utility were controlled for in the regression model. ${ }^{32}$

Incremental cost-effectiveness ratios (ICERs) were calculated from estimated coefficients. To avoid the complications ${ }^{33,34}$ of calculating confidence intervals around ICERs, confidence intervals are presented only for net benefit statistics, which were calculated parametrically from regression output. ${ }^{35}$ Net monetary benefit estimates were calculated using threshold values of $£ 20000$ and $£ 30000$ suggested by the NICE for use within NHS decision- making. ${ }^{15}$ Costs and outcomes were not discounted as trial followup was limited to 12 months. Analysis was conducted using an 'intention to treat' approach.

\section{Sensitivity analysis}

The base-case cost-effectiveness analysis used imputed data. As a sensitivity check, cost-effectiveness was also estimated on complete cases only and the results were compared. For both imputed and complete case data, probabilistic sensitivity analysis was implemented by calculating cost-effectiveness acceptability curves (CEACs) to quantify uncertainty around point estimates of net monetary benefit.

One-way sensitivity analysis was also performed. The sensitivity of the imputed results to self-reported use of secondary care was tested by removing these costs. Although all questionnaire responses concerning secondary care use were checked by a clinician for relevance to depression, the sensitivity analysis provides a means of assessing whether recall bias, misclassification or infrequent but expensive events differed between arms. The sensitivity of the results to the cost of an element of the intervention - that of the BWW licence - was also assessed in order to account for the possibility that these licence costs would be lower if the intervention were rolled out across the NHS.

\section{Results}

A total of 307 participants were randomised to receive the intervention and 302 to the control arm. The mean age in the control (intervention) arm was 50.0 (49.1) years; 68\% (69\%) were female and 97\% (98\%) of participants were of White ethnicity. The mean PHQ-9 score at baseline was 16.7 (17.1), 93\% (91\%) of respondents had previously been treated for depression, and $90 \%$ (87\%) of participants were taking antidepressants at the time of randomisation.

\section{Resource use and cost}

Twenty-six per cent of participants, of those known to have received any calls, received none or little of the intervention (defined as starting two or fewer telephone encounters), 44\% received some of the planned intervention sessions (3-8 encounters) and $29 \%$ of participants received all or almost all of the intervention (9-10 encounters). The median number of encounters was 5 (interquartile range: 2 to 9), and the mean duration of an encounter was $18.5 \mathrm{~min}$.

The mean per participant cost of the intervention was $£ 113$, of which costs associated with telephone encounter calls (such as HIA remuneration and associated on-costs) comprised $66 \%$ of the total (Table 1).

Patients in the intervention arm incurred more primary care costs than did patients in the control arm, primarily because of a greater number of GP consultations. Prescription costs were

Table 1 Mean (s.d.) depression intervention cost (f) per participant for all participants and complete cases

$\begin{array}{lcc}\text { Intervention elements } & \text { All intervention participants }(n=308)^{\mathrm{a}} \text { mean } f(\mathrm{~s} . \mathrm{d} .) & \text { Complete cases }(n=145)^{\mathrm{a}} \text { mean } f(\mathrm{~s} . \mathrm{d} .) \\ \text { Encounter calls } & 71.84(57.64) & 86.55(56.39) \\ \text { Non-scheduled calls } & 3.26(7.17) & 3.58(6.51) \\ \text { All calls } & 75.11(61.04) & 90.13(59.18) \\ \text { LLTTFi website } & 9.74(6.5) & 12.18(6.39) \\ \text { ODLM book } & 9.09(19.98) & 9.45(12.53) \\ \text { Big White Wall } & 19.09(12.75) & 23.88(12.53) \\ \text { Total cost per participant } & 113.03(80.46) & 135.68(77.93)\end{array}$

Total cost per participant

$113.03(80.46)$ 


\begin{tabular}{|c|c|c|c|}
\hline Imputed costs & $N^{a}$ & Usual care mean $\mathrm{f}$ (s.e. $)^{\mathrm{b}}$ & Intervention mean $\mathrm{f}$ (s.e. $)^{\mathrm{b}}$ \\
\hline Imputed mean primary care costs & 609 & $362(15)$ & $404(17)$ \\
\hline Imputed mean drug costs & 609 & $88(12)$ & $92(10)$ \\
\hline Imputed mean hospital, ambulance and other non-primary care NHS costs & 609 & $230(34)$ & $263(37)$ \\
\hline Imputed mean intervention cost & 609 & - & $113(5)$ \\
\hline Imputed mean NHS costs, including the intervention & 609 & $680(41)$ & $872(46)$ \\
\hline Imputed mean PSS costs & 609 & $38(13)$ & $14(5)$ \\
\hline Imputed mean NHS and PSS costs, including the intervention & 609 & $718(45)$ & $886(47)$ \\
\hline
\end{tabular}

similar between arms. Intervention patients incurred higher secondary care costs but lower PSS costs. We use imputed data for our base-case results and because we imputed at the level of aggregate cost categories rather than at the resource level, we present these costs in Table 2. Detailed, disaggregated resource use data on available and complete cases are presented in the supplementary material in Tables DS4 to DS10.

\section{Quality of life}

The QALYs were calculated to adjust for baseline EQ-5D scores (Table 3).The control arm baseline mean EQ-5D score was 0.52 $($ s.e. $=0.02)$ compared with an intervention baseline EQ-5D score of 0.51 (s.e. $=0.02)$, calculated using the imputed data-set.

\section{Cost-consequence results}

Table 4 relates major cost categories to outcomes in a costconsequence matrix, using available data (i.e. before multiple imputation of missing values).

NHS/PSS costs were significantly higher in the intervention arm, largely owing to the cost of the intervention. Participants in the control arm reported higher expenditure on private healthcare costs such as private counselling, psychotherapy, psychiatry and complementary/alternative remedies (mean per patient in available cases: $£ 47.54$ ) than in the intervention arm (mean per patient in available cases: $£ 39.28$ ). Patients also reported slightly higher expenditure in the control arm compared with the intervention arm on out-of-pocket expenses connected with their condition such as self-help books and gym memberships.

The value of lost production was approximately three times higher in the intervention arm for the following reasons. Intervention participants reported a greater number of days affected by depression (mean per patient in available cases: 12.4 working days (s.d. $=42.0)$ in the intervention arm compared with 5.2 working days $($ s.d. $=15.7))$ and more hours taken from work to attend healthcare appointments (mean per patient in available cases: $12.8 \mathrm{~h}(\mathrm{~s} . \mathrm{d} .=86.8)$ in the intervention arm compared with $2.9 \mathrm{~h}$ $($ s.d. $=12.5)$ ).

These findings are influenced by a small number of individuals in the intervention arm who reported large amounts of lost time, and hence these results are probably owing to chance imbalance. No participant in the control arm reported more than 120 working days lost owing to depression, whereas 11 individuals in the intervention arm had at least 120 working days lost, with a mean value among this group of individuals of 187 days.

\begin{tabular}{|c|c|c|c|}
\hline & $N^{a}$ & $\begin{array}{l}\text { Usual care } \\
\text { mean (s.e.) }\end{array}$ & $\begin{array}{l}\text { Intervention } \\
\text { mean (s.e.) }\end{array}$ \\
\hline Imputed adjusted QALYS & 609 & $0.540(0.009)$ & $0.541(0.009)$ \\
\hline
\end{tabular}

The consequences of the intervention include a significant increase in the proportion of responders to treatment, corresponding to a 'number needed to treat' of 12 , and a reduction in mean PHQ- 9 scores of -0.5 (95\% confidence interval -1.5 to 0.5 ).

\section{Cost-effectiveness analysis}

Base-case cost-effectiveness results, using imputed data, from the perspective of a health and social care provider (i.e. NHS/PSS) are presented in Table 5. Results from an NHS-only perspective are presented in online supplementary material (Table DS11 and Fig. DS1) and are similar.

The QALY difference between arms is small (1/1000th of a QALY), equivalent to less than half a day in perfect health, and is associated with incremental costs of $£ 168$. Figure 1 indicates the probability that the intervention is cost-effective at different values of the cost-effectiveness threshold. The intervention is not costeffective at the NICE thresholds of either $£ 20000$ or $£ 30000$.

\section{Sensitivity analyses}

The results are not sensitive to the exclusion of secondary NHS costs. The effect of removing these costs is to slightly narrow the difference in cost between arms relative to the base case, but to leave the modest between-arm QALY difference unchanged. The intervention was not cost-effective under this scenario (Table 6).

Two further deterministic sensitivity analyses were conducted - one in which the cost of BWW licences was reduced by $50 \%$ and another in which the cost of these licences was set to zero. These changes had very minor effects on estimated net benefit and in both cases the intervention was not cost-effective (results not reported).

The cost-effectiveness analysis was re-run on complete cases from an NHS/PSS perspective. In contrast to the analysis on imputed data, this analysis suggested that the intervention is costeffective with a high probability (Table 7). The CEAC for the complete case analysis is presented in the online supplementary material (Fig. DS2).

\section{Discussion}

Analysis of the primary clinical endpoint indicated that the intervention was effective when measured by the pre-specified primary outcome of proportion of responders in each arm measured by PHQ- 9 scores at 4 months. ${ }^{14}$ This improvement is less evident in the 12-month between-arm QALY comparisons and therefore in the overall cost-effectiveness analyses. However, any comparison between the primary clinical outcome and the cost-effectiveness outcome must account for the differences between the outcomes (proportion of responders measured using PHQ-9 and cost-effectiveness using NHS/PSS costs and QALYs), the different time periods over which the outcome is measured (at 4 months and over 12 months) and the overall effect size. 


\begin{tabular}{|c|c|c|c|c|c|}
\hline Available data on costs and consequences & Usual care & $N$ (usual care) & Intervention & $N$ (intervention) & Difference $(95 \% \mathrm{Cl})$ \\
\hline \multicolumn{6}{|l|}{ Costs $(f)$} \\
\hline Mean cost of intervention & $0^{\frac{\hbar}{*}}$ & 188 & 136 & 169 & - \\
\hline $\begin{array}{l}\text { Mean cost of NHS resources, excluding } \\
\text { cost of intervention }\end{array}$ & 645 & 188 & 709 & 169 & $64(-76 \text { to } 193)^{a}$ \\
\hline Mean cost of NHS resources, including intervention & 646 & 188 & 845 & 169 & $199(79 \text { to } 339)^{\mathrm{a}}$ \\
\hline Mean cost of PSS & 37 & 193 & 15 & 171 & $-21(-73 \text { to } 1)^{a}$ \\
\hline $\begin{array}{l}\text { Mean cost of NHS and PSS resources, } \\
\text { including intervention }\end{array}$ & 683 & 188 & 860 & 169 & $177(41 \text { to } 317)^{a}$ \\
\hline Out-of-pocket expenses & 199 & 246 & 177 & 233 & $-21(-116 \text { to } 57)^{a}$ \\
\hline $\begin{array}{l}\text { Mean societal value per patient of } \\
\text { lost production }\end{array}$ & 74 & 246 & 242 & 233 & $168(45 \text { to } 362)^{a}$ \\
\hline \multicolumn{6}{|l|}{ Consequences $^{\mathrm{b}}$} \\
\hline $\begin{array}{l}\text { PHQ-9 response at } 4 \text { months, adjusted for } \\
\text { site and baseline PHQ-9 }\end{array}$ & $19 \%$ & 270 & $27 \%$ & 255 & Odds ratio 1.7 (1.1 to 2.5 ) \\
\hline Adjusted mean PHQ-9c & 12.0 & 261 & 11.5 & 255 & $-0.5(-1.5$ to 0.5$)$ \\
\hline$E Q-5 D-5 L^{d}$ & 0.564 & 227 & 0.569 & 219 & $0.005(-0.053 \text { to } 0.061)^{\mathrm{a}}$ \\
\hline Adjusted QALYS ${ }^{d}$ & 0.536 & 175 & 0.567 & 158 & $0.031(-0.022 \text { to } 0.0810)^{\mathrm{a}}$ \\
\hline \multicolumn{6}{|c|}{$\begin{array}{l}\mathrm{Cl} \text {, confidence interval; PSS, personal social services; PHQ-9, Patient Health Questionnaire; QALYS, quality-adjusted life years. Costs are reported accurate to } £ 1 \text { and may not sum to } \\
\text { apparent totals owing to the effects of rounding. } \\
\ddagger \text { One participant in the control arm received elements of the intervention, the estimated cost of which was estimated to be } £ 0.38 \text {. } \\
\text { a. Confidence interval calculated as accelerated and bias corrected interval from } 1000 \text { bootstrap replicates to account for the skewed distribution of costs. } \\
\text { b. Except where otherwise stated, all consequences were measured at } 12 \text { months, or over a period up to } 12 \text { months. } \\
\text { c. Measured as the adjusted mean difference in continuous PHQ-9 scores at } 12 \text { months. } \\
\text { d. Based on available data, and adjusted for baseline EQ-5D responses. }\end{array}$} \\
\hline
\end{tabular}

\section{Table 5 Cost-effectiveness of the Healthlines intervention from an NHS/PSS perspective}

\begin{tabular}{|c|c|c|c|}
\hline & Usual care mean & Intervention mean & Difference $(95 \% \mathrm{Cl})$ \\
\hline \multicolumn{4}{|l|}{ Costs and QALYS } \\
\hline Total NHS and PSS costs & $£ 718$ & f886 & $£ 168$ (£43 to $£ 294)$ \\
\hline QALYS & 0.540 & 0.541 & $0.001(-0.023$ to 0.026$)$ \\
\hline \multicolumn{4}{|l|}{ Cost-effectiveness statistics } \\
\hline \multicolumn{4}{|l|}{ ICER: $£ 132630$} \\
\hline \multicolumn{4}{|c|}{ Probability that intervention cost-effective at CE threshold of $f 20$ 000: 0.30} \\
\hline \multicolumn{4}{|c|}{ Probability that intervention cost-effective at CE threshold of $£ 30$ 000: 0.37} \\
\hline \multicolumn{4}{|c|}{ NMB at threshold of $£ 20000$ (95\% confidence interval): $-£ 143$ (-£164 to -122) } \\
\hline \multicolumn{4}{|c|}{$\begin{array}{l}\mathrm{Cl} \text {, confidence interval; PSS, personal social services; QALYS, quality-adjusted life years; ICER, incremental cost-effectiveness ratio; CE, COSt-effectiveness; NMB, net monetary benefit. } \\
\text { We report confidence intervals for the point estimate of net benefit, but not for the ICER. Confidence intervals for the ICER can be both difficult to interpret }{ }^{34} \text { and statistically } \\
\text { intractable. Instead, we place an emphasis throughout our analysis on net benefit. We present cost-effectiveness acceptability curves and confidence intervals around net benefit } \\
\text { to represent uncertainty in our cost-effectiveness results. }\end{array}$} \\
\hline
\end{tabular}

Three considerations are important in interpreting the small between-arm QALY difference. The first is that these differences are consistent with the modest mean effect size in analysis of the intervention on the primary PHQ-9 clinical outcome and the possibility that the intervention was helpful for some but not all

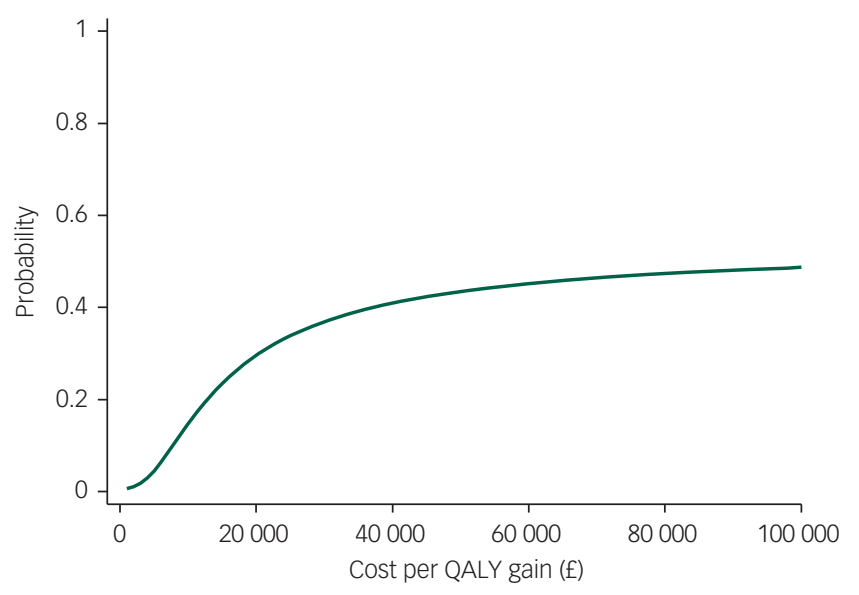

Fig. 1 Cost-effectiveness acceptability curve from an NHS/PSS perspective for imputed model. participants. ${ }^{14}$ Analysis of secondary trial outcomes indicated greater improvements in anxiety, and greater satisfaction with support, among those randomised to the intervention. ${ }^{14}$

The second consideration is that analysis of correlation (using Spearman's rho) indicates that high PHQ-9 scores (indicating greater severity of depression) and low EQ-5D scores (indicating lower quality of life) are correlated at baseline and all followup time points. The null of independence was rejected in each case $(P<0.001)$, and the absolute value of the correlations were largest with the anxiety/depression domain of the EQ-5D instrument. This is tentative evidence that the two instruments indicate the same 'direction of travel' for outcomes, and that the lower sensitivity ${ }^{21}$ of the EQ-5D instrument used in the QALY calculations is not as important to the analysis as the finding that the intervention exerted a positive but modest impact on depression and particularly on health-related quality of life.

The third consideration relates to the amount of missing data. Some $51 \%$ of participants had incomplete data on variables necessary to conduct an inferential cost-effectiveness analysis. This was in spite of near-complete data on primary care resource use, prescriptions and intervention cost. Incomplete questionnaire responses to questions on quality of life and NHS resource use account for almost all of the missing data. This was largely 


\begin{tabular}{|c|c|c|c|}
\hline & Usual care mean & Intervention mean & Difference $(95 \% \mathrm{Cl})$ \\
\hline \multicolumn{4}{|l|}{ Costs and QALYS } \\
\hline Total NHS/PSS costs & $£ 488$ & f623 & $£ 135$ ( $£ 70$ to $£ 199$ ) \\
\hline QALYS & 0.540 & 0.541 & $0.001(-0.023$ to 0.026$)$ \\
\hline \multicolumn{4}{|c|}{ Cost-effectiveness statistics } \\
\hline \multicolumn{4}{|l|}{ ICER: $£ 114624$} \\
\hline \multicolumn{4}{|c|}{ Probability that intervention cost-effective at CE threshold of $£ 20000: 0.33$} \\
\hline \multicolumn{4}{|c|}{ Probability that intervention cost-effective at CE threshold of $£ 30$ 000: 0.40} \\
\hline \multicolumn{4}{|c|}{ NMB at threshold of $£ 20000$ (95\% confidence interval): $-£ 111$ (-£132 to -91) } \\
\hline \multicolumn{4}{|c|}{$\begin{array}{l}\mathrm{Cl} \text {, confidence interval; PSS, personal social services; QALYS, quality-adjusted life years; ICER, incremental cost-effectiveness ratio; CE, cost-effectiveness; NMB, net monetary benefit. } \\
\text { We report confidence intervals for the point estimate of net benefit, but not for the ICER. Confidence intervals for the ICER can be both difficult to interpret }{ }^{34} \text { and statistically } \\
\text { intractable. } \text { Instead, we place an emphasis throughout our analysis on net benefit. We present cost-effectiveness acceptability curves and confidence intervals around net benefit }^{33} \text { to represent uncertainty in our cost-effectiveness results. }\end{array}$} \\
\hline
\end{tabular}

\begin{tabular}{|c|c|c|c|}
\hline & Usual care mean $(n=155)$ & Intervention mean ( $n=144)$ & Difference $(95 \% \mathrm{Cl})$ \\
\hline \multicolumn{4}{|l|}{ Costs and QALYS } \\
\hline Total NHS/PSS costs - complete case & £719 & f864 & $£ 145(-f 11$ to $£ 300)$ \\
\hline QALYS - complete case & 0.535 & 0.573 & 0.037 (0.009 to 0.066$)$ \\
\hline \multicolumn{4}{|l|}{ Cost-effectiveness statistics } \\
\hline \multicolumn{4}{|l|}{ ICER: £3850 } \\
\hline \multicolumn{4}{|c|}{ Probability that intervention cost-effective at CE threshold of $£ 20000: 0.98$} \\
\hline \multicolumn{4}{|c|}{ Probability that intervention cost-effective at CE threshold of $£ 30$ 000: 0.99} \\
\hline \multicolumn{4}{|c|}{ NMB at threshold of 20000 (95\% Confidence interval): $£ 607$ ( $£ 572$ to 642 ) } \\
\hline
\end{tabular}

because of licencing restrictions imposed on the use of the EQ-5D questionnaire, which had to be sent separately from the main follow-up questionnaires, and had to be sent by post when most outcomes were collected online.

The incremental costs of the intervention arm did not change drastically between imputed cases, available cases and complete cases, largely because of near-complete data on the intervention (the major element of intervention arm costs) and on primary care data. The estimated QALYs from the imputed dataset, adjusting for baseline differences, differed by 0.001 between groups, compared with 0.037 QALYs in complete cases. Below, we explain why the complete case analysis is likely to be both biased and inefficient.

\section{Strengths}

The Healthlines RCT was designed as a pragmatic, theory-based intervention to support patients with a prevalent long-term condition. To our knowledge, it is one of the largest RCTs conducted of a complex intervention based on telehealth for patients with depression. The design and implementation of the intervention itself was the evidence-based culmination of a broad body of work intended to support the development of a responsive, flexible telehealth service.

This economic evaluation adds to the limited evidence base on the cost-effectiveness of telehealth ${ }^{37}$ and internet interventions for mental health. ${ }^{38}$ The economic evaluation was based on analysis of extensive and detailed patient-level data and, unlike a number of studies included in the systematic review of Mistry et al, ${ }^{37}$ was conducted with reference to guidelines for best practice in economic evaluation. ${ }^{39}$

\section{Limitations}

The amount of missing cost and quality of life data poses challenges for the analysis and interpretation of results. We attempted to moderate any undue influence of the specific imputation model implemented by following recommended practice in multiple imputation. We compared results of available, complete and imputed cases to assess the reasonableness of the outputs from the imputation model.

It is notable that there was a marked difference between the results using imputed data or complete cases. Estimated NHS costs are similar between complete, imputed and available cases.

\begin{tabular}{|c|c|c|c|c|}
\hline \multirow[b]{2}{*}{ Quality of life } & \multicolumn{2}{|c|}{ Usual care } & \multicolumn{2}{|c|}{ Intervention } \\
\hline & Mean & N & Mean & N \\
\hline \multicolumn{5}{|l|}{ Baseline } \\
\hline All available data & 0.52 & 268 & 0.51 & 273 \\
\hline Complete cases & 0.52 & 155 & 0.54 & 144 \\
\hline Non-complete cases ${ }^{a}$ & 0.53 & 113 & 0.49 & 129 \\
\hline \multicolumn{5}{|l|}{ 4-month follow-up } \\
\hline All available data & 0.53 & 233 & 0.56 & 220 \\
\hline Complete cases & 0.52 & 155 & 0.59 & 144 \\
\hline Non-complete cases ${ }^{a}$ & 0.56 & 78 & 0.50 & 76 \\
\hline \multicolumn{5}{|l|}{ 8-month follow-up } \\
\hline All available data & 0.54 & 227 & 0.56 & 210 \\
\hline Complete cases & 0.53 & 155 & 0.59 & 144 \\
\hline Non-complete cases ${ }^{a}$ & 0.57 & 72 & 0.48 & 66 \\
\hline \multicolumn{5}{|l|}{ 12-month follow-up } \\
\hline All available data & 0.57 & 225 & 0.57 & 218 \\
\hline Complete cases & 0.56 & 155 & 0.58 & 144 \\
\hline Non-complete cases ${ }^{a}$ & 0.60 & 70 & 0.56 & 74 \\
\hline
\end{tabular}

a. Non-complete cases in this table refer to participants who did not have complete EQ-5D data at all time points. The number of available observations at each time point is therefore the sum of complete and non-complete cases. 
The pattern of missing EQ-5D data between different cases is summarised in Table 8.

Complete case analysis is likely to be inefficient because it would discard data from half of all trial participants. This analysis would be biased because missingness in quality of life data seems to be conditional on observed allocation to the intervention arm, since participants in the intervention who did not provide data at all time points had, on average, lower quality of life than those who provided complete data (Table 8). In the control group, the reverse is true: non-responders to the questionnaires had, on average, a higher quality of life than the complete cases. Table 8 suggests a mechanism for the complete and imputed QALYs to differ.

The amount of missingness in quality of life data complicates comparisons with other trials. For example, the economic evaluation $^{40}$ of the CADET primary care trial of collaborative care, ${ }^{41}$ involving structured and scheduled patient follow-ups and enhanced communication between medical professionals, determined that the intervention was likely to be cost-effective. An important difference between the studies is the amount of missing data in each trial, with CADET reporting missingness of up to $25 \%$ compared with $51 \%$ in Healthlines.

The level of adherence to the intervention is another potential limitation. The median number of telephone encounters known to have been initiated was 5 , out of a total of 10 scheduled encounters, although a focus on the number of completed telephone encounters does not reflect all aspects of compliance with the intervention. There is a lack of methodological guidance concerning how adherence should be reflected in economic evaluation, ${ }^{42}$ albeit the focus on actual adherence in the base-case analysis reflects the pragmatic design of the trial. A complier-average causal effect analysis, using the principal stratification method, of the main trial results ${ }^{13}$ suggested greater effectiveness among participants who received more telephone encounters, although this did not account for baseline variables.

Other limitations include the trial follow-up period, which was limited to 12 months. Longer-term follow-up would be necessary to establish persistence of effect and whether ongoing versus timelimited telehealth support would be most appropriate for this patient group.

It is not clear how closely the operation of the Healthlines service would reflect a system-wide implementation. In practice, scale effects and alternative rostering of HIAs and scheduling of calls may secure more efficient operation, although there is no evidence from the trial itself to indicate that substantial efficiency improvements were available but left unexploited.

In order for the intervention to be cost-effective, it is likely that it would need to be more effective rather than less costly. Holding incremental costs constant, and ignoring the effects of uncertainty, the threshold incremental QALY difference necessary to result in an ICER $<£ 20000$ is approximately 0.085 or about eight times the effect size actually observed in the trial. Improving the effectiveness of the intervention is likely to require better targeting of the intervention to those interested in using it, efforts to improve patient engagement and more effective optimisation of anti-depressant medication when patients fail to respond to treatment. ${ }^{14}$

In conclusion, the Healthlines service was found to be acceptable to patients as a means of condition management, ${ }^{18}$ and response to treatment after 4 months was higher for participants randomised to the intervention. ${ }^{14}$ However, the intervention was associated with a small incremental QALY gain at 12 months and was not likely to be cost-effective at a threshold value of $£ 20000$.

\begin{abstract}
Padraig Dixon, DPhil, Centre for Academic Primary Care, School of Social and Community Medicine, University of Bristol, Bristol, UK; Sandra Hollinghurst, PhD, Centre for Academic Primary Care, School of Social and Community Medicine, University of Bristol, Bristol, UK; Louisa Edwards, PhD, Centre for Academic Primary Care, School of Social and Community Medicine, University of Bristol, Bristol, UK: Clare Thomas, PhD, Centre for Academic Primary Care, School of Social and Community Medicine, University of Bristol, Bristol, UK; Alexis Foster, MPH, Clinical Trials Research Unit, School of Health and Related Research (ScHARR), University of Sheffield, Sheffield, UK; Ben Davies, PhD, Centre for Academic Primary Care, School of Social and Community Medicine, University of Bristol, Bristol, UK; Daisy Gaunt, MSC, Bristol Randomised Trials Collaboration (BRTC), School of Social and Community Medicine, University of Bristol, Bristol, UK; Alan A. Montgomery, PhD, Bristol Randomised Trials Collaboration (BRTC), School of Social and Community Medicine, University of Bristol, Bristol, UK; Nottingham Clinical Trials Unit, Faculty of Medicine \& Health Sciences, Queen's Medical Centre, University of Nottingham, Nottingham, UK; Chris Salisbury MD, Centre for Academic Primary Care, School of Social and Community Medicine, University of Bristol, Bristol, UK
\end{abstract}

Correspondence: Padraig Dixon, Centre for Academic Primary Care, School of Socia and Community Medicine, University of Bristol, Canynge Hall, 39 Whatley Road, Bristo BS8 2PS, UK. Email: Padraig.Dixon@bristol.ac.uk

First received 8 Mar 2016, revised 1 Jul 2016, accepted 1 Jul 2016

\section{Acknowledgements}

We are grateful to all patients, healthcare professionals, health information advisors and other NHS Direct staff who contributed time and effort to make the Healthlines trial possible. We thank administrative staff at trial sites for support with participant recruitment, data entry and trial administration. The Healthlines study was designed and delivered in collaboration with Bristol Randomised Trials Collaboration (BRTC), a UKCRC Registered Clinical Trials Unit in receipt of National Institute for Health Research CTU support funding.

\section{Funding}

This report summarises independent research funded by the National Institute for Health Research (NIHR) under its Programme Grant for Applied Research (Grant Reference Number RP-PG-0108-10011). The views and opinions expressed in this report are those of the authors and do not necessarily reflect those of the National Institute for Health Research (NIHR), the NHS or the Department of Health. The funder had no role in the conduct of the study, the writing of the manuscript or the decision to submit it for publication.

\section{References}

1 Department of Health. Long Term Conditions Compendium of Information. Department of Health, 2012.

2 Kessler RC, Aguilar-Gaxiola S, Alonso J, Chatterji S, Lee S, Ormel J, et al. The global burden of mental disorders: an update from the WHO World Mental Health (WMH) Surveys. Epidemiol Psichiatr Soc 2009; 18: 23-33.

3 Hardeveld F, Spijker J, De Graaf R, Nolen WA, Beekman ATF. Prevalence and predictors of recurrence of major depressive disorder in the adult population. Acta Psychiatr Scand 2010; 122: 184-91.

4 Kessler RC. The costs of depression. Psychiatr Clin North Am 2012; 35: 1-14.

5 London School of Economics and Political Science Centre for Economic Performance Mental Health Policy Group. The Depression Report: A New Deal for Depression and Anxiety Disorders. LSE Research Online, 2006.

6 Vassilev I, Rowsell A, Pope C, Kennedy A, O'Cathain A, Salisbury C, et al. Assessing the implementability of telehealth interventions for self-management support: a realist review. Implement Sci 2015; 10: 59.

7 World Health Organization. Glossary of Globalization, Trade and Health Terms. Secondary Glossary of Globalization, Trade and Health Terms (http://www.who. int/trade/glossary/story021/en/).

8 Wittkampf KA, Naeije L, Schene AH, Huyser J, van Weert HC. Diagnostic accuracy of the mood module of the Patient Health Questionnaire: a systematic review. Gen Hosp Psychiatry 2007; 29: 388-95.

9 Kendrick T, Dowrick C, McBride A, Howe A, Clarke P, Maisey S, et al. Management of depression in UK general practice in relation to scores on depression severity questionnaires: analysis of medical record data. BMJ 2009; 338: b750.

10 Kroenke K, Spitzer RL, Williams JBW. The PHQ-9. J Gen Intern Med 2001; 16 606-13.

11 Salisbury C, Thomas C, O'Cathain A, Rogers A, Pope C, Yardley L, et al. TElehealth in CHronic disease: mixed-methods study to develop the TECH 
conceptual model for intervention design and evaluation. BMJ Open 2015; 5 e006448.

12 Thomas CL, Man M-S, O'Cathain A, Hollinghurst S, Large S, Edwards L, et al. Effectiveness and cost-effectiveness of a telehealth intervention to support the management of long-term conditions: study protocol for two linked randomized controlled trials. Trials 2014; 15: 36.

13 Salisbury C, O'Cathain A, Thomas C, Edwards L, Montgomery AA, Hollinghurst S, et al. Telehealth for patients with long-term health conditions: development and evaluation of the Healthlines Service. NIHR J Libr Programme Grants Appl Res, in press

14 Salisbury C, O'Cathain A, Edwards L, Thomas C, Gaunt D, Hollinghurst S, et al. Effectiveness of an integrated telehealth service for patients with depression: a pragmatic randomised controlled trial of a complex intervention. Lancet Psychiatry 2016; 3: 515-25.

15 NICE. Guide to the Methods of Technology Appraisal. 2013

16 Merriel SW, Andrews V, Salisbury C. Telehealth interventions for primary prevention of cardiovascular disease: a systematic review and meta-analysis. Prev Med 2014; 64: 88-95.

17 Segar J, Rogers A, Salisbury C, Thomas C. Roles and identities in transition: boundaries of work and inter-professional relationships at the interface between telehealth and primary care. Health Soc Care Community 2013; 21: 606-13.

18 Edwards L, Thomas C, Gregory A, Yardley L, O'Cathain A, Montgomery AA, et al. Are people with chronic diseases interested in using telehealth? A cross-sectiona postal survey. J Med Internet Res 2014; 16: e123.

19 Herdman M, Gudex C, Lloyd A, Janssen MF, Kind P, Parkin D, et al. Development and preliminary testing of the new five-level version of EQ-5D (EQ-5D-5L). Qual Life Res 2011; 20: 1727-36.

20 Mihalopoulos C, Chen G, lezzi A, Khan MA, Richardson J. Assessing outcomes for cost-utility analysis in depression: comparison of five multi-attribute utility instruments with two depression-specific outcome measures. Br J Psychiatry 2014; 205: 390-7.

21 Brazier J, Connell J, Papaioannou D, Mukuria C, Mulhern B, Peasgood T, et al. A systematic review, psychometric analysis and qualitative assessment of generic preference-based measures of health in mental health populations and the estimation of mapping functions from widely used specific measures. Health Technol Assess 2014; 18(34)

22 Curtis L. Unit Costs of Health and Social Care 2013. Personal Social Services Research Unit, 2013.

23 Department of Health. Reference Costs 2012/13. Department of Health, 2013.

24 Prescribing and Primary Care Team, Health and Social Care Information Centre Prescription Cost Analysis: England 2013 (available from http://www.hscic.gov.uk/ catalogue/PUB13887). Health and Social Care Information Centre, 2014.

25 ONS. Annual Survey of Hours and Earnings, 2013 Provisional Results. ONS, 2013.

26 Royston P, White IR. Multiple Imputation by Chained Equations (MICE): implementation in Stata. J Stat Softw 2011; 45: 1-20.

27 White $\mathbb{R}$, Royston $\mathrm{P}$, Wood AM. Multiple imputation using chained equations: issues and guidance for practice. Stat Med 2011; 30: 377-99.
28 Eurogol Interim scoring for the EQ-5D-5L: Mapping the EQ-5D-5L to EQ-5D-3L value sets. Secondary Interim scoring for the EQ-5D-5L: Mapping the EQ-5D-5L to EQ-5D-3L value sets (http://www.euroqol.org/fileadmin/user upload/Documenten/ PDF/Crosswalk_5L/EQ-5D-5L_Crosswalk_model_and_methodology.pdf).

29 Glick H, Doshi J, Sonnad S, Polsky D. Economic Evaluation in Clinical Trials. Oxford University Press, 2007.

30 Faria $\mathrm{R}$, Gomes $\mathrm{M}$, Epstein $\mathrm{D}$, White I. A guide to handling missing data in costeffectiveness analysis conducted within randomised controlled trials. Pharmacoeconomics 2014; 32: 1157-70.

31 Rubin DB. Introduction. Multiple Imputation for Nonresponse in Surveys: 1-26. Wiley, 1987

32 Manca A, Hawkins N, Sculpher MJ. Estimating mean QALYs in trial-based costeffectiveness analysis: the importance of controlling for baseline utility. Health Econ 2005; 14: 487-96.

33 Drummond $\mathrm{M}$, Sculpher $\mathrm{M}$, Claxton $\mathrm{K}$, Stoddart G, Torrance G. Methods for the Economic Evaluation of Health Care Programmes. 4th ed. Oxford University Press, 2015

34 Briggs $\mathrm{AH}$. Handling uncertainty in cost-effectiveness models. Pharmacoeconomics 2000; 17: 479-500.

35 Gray A, Clarke PM, Wolstenholme JL, et al. Applied Methods of Cost-effectiveness Analysis in Healthcare. Oxford University Press, 2010.

36 Williams C. Overcoming Depression and Low Mood: A Five Areas Approach. 3rd ed. Hodder Arnold, 2009.

37 Mistry H, Garnvwa H, Oppong R. Critical appraisal of published systematic reviews assessing the cost-effectiveness of telemedicine studies. Telemed I E Health 2014; 20: 609-18

38 Donker T, Blankers M, Hedman E, Ljotsson B, Petrie K, Christensen H. Economic evaluations of Internet interventions for mental health: a systematic review. Psychol Med 2015; 45: 3357-76.

39 Husereau D, Drummond M, Petrou S, Carswell C, Moher D, Greenberg D, et al. Consolidated Health Economic Evaluation Reporting Standards (CHEERS) statement BMJ 2013; 346: f1049.

40 Green C, Richards DA, Hill J, Gask L, Lovell K, Chew-Graham C et al. Costeffectiveness of collaborative care for depression in UK primary care: economic evaluation of a randomised controlled trial (CADET). PLOS One 2014; 9: e104225.

41 Richards DA, Hill JJ, Gask L, Lovell K, Chew-Graham C, Bower P, et al. Clinical effectiveness of collaborative care for depression in UK primary care (CADET): cluster randomised controlled trial. BMJ 2013; 347: f4913.

42 Brilleman SL, Metcalfe C, Peters TJ, Hollingworth W. The reporting of treatment nonadherence and its associated impact on economic evaluations conducted alongside randomized trials: a systematic review. Value Health 2016; 19: 99-108.

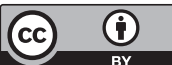

